\title{
nutritionDay 2020 en Colombia, un encuentro con la realidad COVID
}

\author{
nutritionDay 2020 in Colombia, a meeting with the COVID reality
}

Dia da Nutrição 2020 na Colômbia, um encontro com a realidade COVID

\author{
Angélica María Pérez Cano ${ }^{1 *}$
}

https://doi.org/10.35454/rncm.v4n4.355

\section{INTRODUCCIÓN}

El 2020 realmente fue un desafío para todos los que ejercemos nutrición clínica: la sobrecarga laboral, el aumento de pacientes con soporte nutricional con enfermedad por coronavirus de 2019 (COVID-19), el afán de que los pacientes se beneficiaran de la atención nutricional y que pudiéramos proveer el mejor cuidado fueron los desafíos que tuvimos que superar estos meses de pandemia. A pesar de estos desafíos, no dejamos de participar en el nutritionDay.

La participación de las instituciones en el nutritionDay 2020 permitió mostrar cómo se realiza el proceso de cuidado nutricional durante la pandemia; esto incluye los factores de riesgo de desnutrición, las estructuras de atención y la atención nutricional. Los datos de cada institución se comparan con los del país y la región, y con los datos internacionales. Esta información y la probabilidad de comparase con otras instituciones proporcionan información de base para el análisis y definición de planes de acción institucionales. Es importante aclarar que esta información no implica evidencia científica de efectividad del cuidado nutricional. Para ello es necesario realizar otro tipo de análisis, como lo han hecho anteriormente investigadores de la Asociación Colombiana de Nutrición Clínica (ACNC) y de otros países ${ }^{(1,2)}$.

Coordinadora nacional, Colombia nutritionDay. Jefe del departamento de nutrición, Hospital Universitario Infantil de San José. Presidente de la Asociación Colombiana de Nutrición Clínica (ACNC).

*Correspondencia: Angélica María Pérez Cano.

nutritionday@nutriclinicacolombia.org
En el contexto de pandemia, más de la mitad de las instituciones que participaron en años anteriores no pudo hacerlo en esta ocasión. A continuación les presentamos los resultados de 15 instituciones, 27 unidades y 801 pacientes; por un lado, las instituciones que participaron lo han venido haciendo desde hace varios años $y$ han venido implementando y mejorando el proceso de cuidado nutricional (es por esta razón que los datos de este año son distintos: son el reflejo de instituciones comprometidas con el nutritionDay); y, por otro lado, los pacientes de COVID-19 fueron remitidos más rápido a las unidades de nutrición, lo que aumenta las cifras de intervención nutricional.

Es importante resaltar que el $100 \%$ de las instituciones cuenta con estrategias de cuidado nutricional, cifra superior al $80 \%$ de la referencia internacional. Nos alegra pensar que la ACNC ha podido concientizar a estas unidades sobre la importancia de empoderar a los departamentos de nutrición clínica y soporte nutricional.

Los datos del nutritionDay 2020 de Colombia permiten observar que hay una cantidad aproximada de un nutricionista por cada 58 pacientes y, en el $63 \%$ de las unidades participantes, existe un grupo de soporte nutricional. Por tanto, seguimos teniendo déficit en la cantidad de nutricionistas y equipos de soporte nutricional para la atención oportuna de nuestros pacientes. Consideramos que es imperativo lograr una cantidad mayor de profesionales en nutrición por cantidad de pacientes.

El 85,1 \% de las unidades o servicios hospitalarios realiza tamizaje nutricional a los pacientes al ingreso, con un incremento interesante con respecto a años anteriores. Las herramientas más usadas son Malnutrition Screening Tool (MST), Nutrition risk score 2002 (NRS 2002) y 
Malnutrition universal screening tool (MUST). En el día del nutritionDay se determinó un riesgo nutricional en el 44,4 \% de los pacientes. En la práctica habitual de las unidades, el $70 \%$ de los pacientes desnutridos y el $63 \%$ los pacientes en riesgo tienen una intervención nutricional. El $74,1 \%$ de los pacientes se pesa al ingreso. Se identifica que en más del $60 \%$ de los pacientes se realizan intervenciones que permiten garantizarles una alimentación adecuada.

En cuanto a la calidad de la atención, un 70,4 \% de las instituciones solamente maneja indicadores de calidad, pero no se realizan reportes de estos indicadores al área de calidad; solamente el 40,7 \% genera reportes de gestión a los directivos del hospital. En el 85,2 \% de las instituciones se tiene acceso a la historia clínica para indicar si un paciente está en riesgo nutricional o padece desnutrición durante su hospitalización; pero al momento del alta, solamente el 22,2 \% resume el tratamiento nutricional recibido durante la estadía; $\sin$ embargo, al 70,4\% de los pacientes se les entregan recomendaciones nutricionales de egreso.

En los 801 pacientes se encontró una edad promedio de 59 años, con un $49 \%$ de pacientes de sexo femenino y un promedio de índice de masa corporal (IMC) de 24,9 . El tipo de seguridad social en salud sigue siendo mayor a nivel público, con un $61,6 \%$, que privado, con un $17,4 \%$. En el $76,8 \%$ de casos hubo hospitalización derivada de una urgencia, las primeras causas diagnósticas al ingreso fueron por enfermedades del sistema circulatorio y respiratorio, y enfermedades oncológicas; con una comorbilidad encontrada en su mayoría con diabetes mellitus, infección e insuficiencia cardíaca. En cuanto a la COVID-19; el 10,6 \% de la muestra tenía diagnóstico positivo de esta patología; el 15,4 \% había tenido resultado positivo para COVID-19 en el último año y, de ellos, el 14,5 \% requirió hospitalización previa.

Se encontró en la población total un $14,9 \%$ con desnutrición y un $26,9 \%$ con riesgo de desnutrición. En cuanto a la implementación de la terapia nutricional, hubo un 44,6 \% de los pacientes con menú normal del hospital, un $11 \%$ con suplementación nutricional oral, un $7 \%$ con nutrición enteral, un 4,6\% con nutrición parenteral y un $34,2 \%$ con dieta especial o terapéutica. En las actividades realizadas desde el ingreso hospitalario se encuentra un $38,6 \%$ de instituciones donde se determinan los requerimientos nutricionales, un $45,9 \%$ desarrolla un plan de tratamiento nutricional y un $45,9 \%$ consulta con un experto en nutrición. Dentro de la monitorización y seguimiento del plan nutricional, en promedio se tienen dietas entre 1500 y 1999 calorías en las instituciones como objetivo calórico, aunque un $42,5 \%$ no determina estos objetivos dentro de sus actividades. La percepción de los pacientes indica que en un 70,6\% ha mejorado su estado de salud, en un 7,7 \% se ha deteriorado y en un $18,2 \%$ no ha cambiado su estado. Los días de estancia hospitalaria aumentaron este año con un promedio de 16 días. En cuanto a los desenlaces a los 30 días de la primera recolección de datos, el 14,2 \% de los pacientes continúa hospitalizado, el 73,3\% fue dado de alta, el 2,8\% fue transferido a otro hospital y se encontró una mortalidad aumentada del $6,3 \%$ y un porcentaje de readmisión del 6,9\%.

Con respecto a los hábitos alimentarios habituales de los pacientes del estudio, el 20,8 \% indicó que evita azúcares añadidos; el 14,5\%, que sigue una dieta baja en grasas, y el 6,7\%, intolerancia a la lactosa. En cuanto a la capacidad funcional, el 25,9\% necesitó ayuda para caminar y el $15,8 \%$ estuvo postrado en cama. Se encontró polifarmacia en un $27,9 \%$ de los pacientes ( 3 a 5 medicamentos al día). Al preguntar sobre la pérdida de peso, el 49,2\% perdió peso involuntariamente en los últimos 3 meses; y en cuanto a ingesta, el 31,6\% la disminuyó en la última semana. Sorprendentemente, el $55 \%$ requirió asistencia para alimentarse, el 40,9\% de los pacientes no consumió totalmente la alimentación del hospital y el 14,8 \% no consumió nada; y dentro de las razones por las cuales no comieron todo en el hospital se encontró que no les gustó el tipo de comida ofrecida, no tenían apetito habitual, no les gustó el sabor o el olor de la comida, o tuvieron náuseas o vómito; esto produjo que el 14,6\% de los pacientes recibiera comidas de fuera del hospital (de preferencia, frutas, alimentos dulces y salados). Al indagar sobre el apetito en general, el 37,4 \% indicó que había disminuido.

En el marco del mejoramiento continuo de la calidad, la ACNC invita a las instituciones a defender los derechos a la alimentación y a la salud, y promover la lucha contra la malnutrición, recordando los 13 principios de la Declaración de Cartagena ${ }^{(3)}$ que constituyen el fundamento para promover el desarrollo del cuidado nutricional en el ámbito clínico. 


\section{Indicadores de calidad de la atención nutricional}

NutritionDay 2020

\section{\IDENTIFICACIÓN \\ Paso DE RIESGO}

\section{Paso 02 DIAGNÓSTICO
NUTRICIONAL}

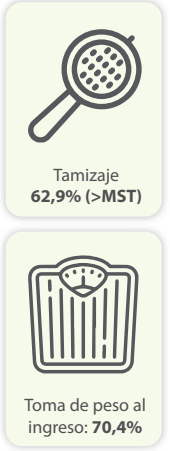

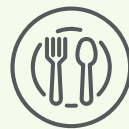

Riesgo nutricional: $26,9 \%$

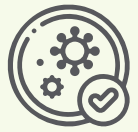

COVID-19 positivo: $10,6 \%$

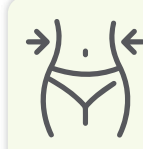

Pérdida de peso

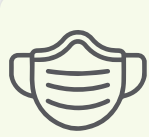

Post COVID-19:

15,4
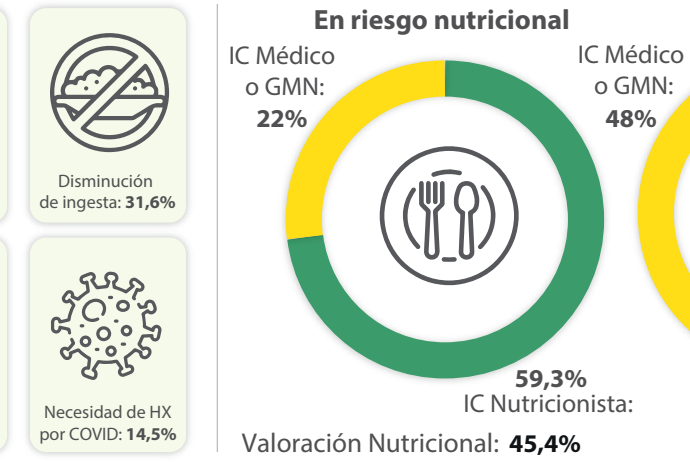

Malnutridos

Valoración Nutricional: $\mathbf{4 5 , 4 \%}$

\section{Paso 2 INTERVEnCIÓN}

\section{Paso J4. MONITOREO Y SEGUIMIENTO}

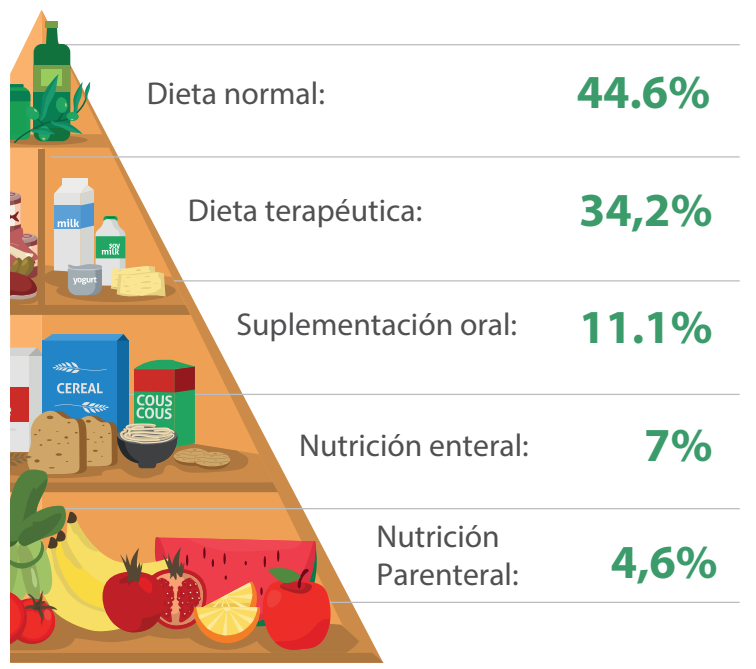

Manejo de indicadores en PCN: $\mathbf{7 0 , 4 \%}$

Estancia hospitalaria promedio: 16 días

D

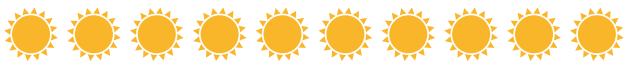

El $40,9 \%$ de los pacientes no consumieron todo

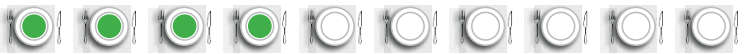

\section{Platos según consumo:}

Todo: $55,3 \quad$ Mitad: $17,3 \quad$ 1/4: $8,8 \quad$ Nada: $14,8 \%$

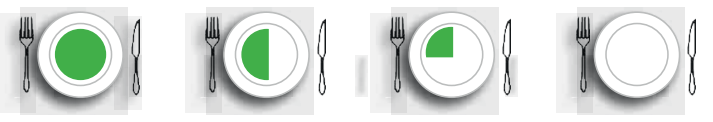

\section{Paso DE MALEJO NUTRICIONAL}

\section{Paso OP ALGUIMIENTO}

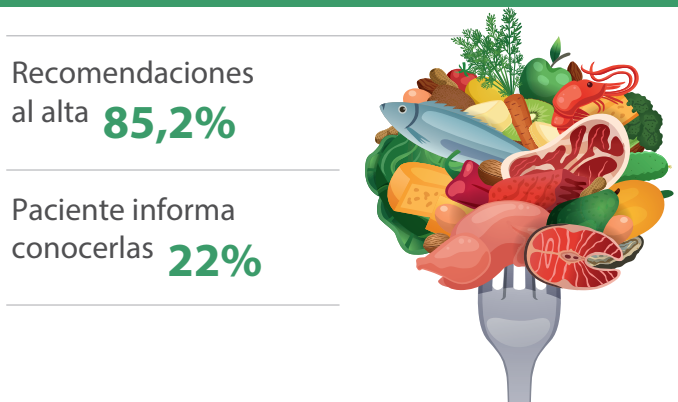

Reingreso: $10 \%$

Mortalidad: 6,3

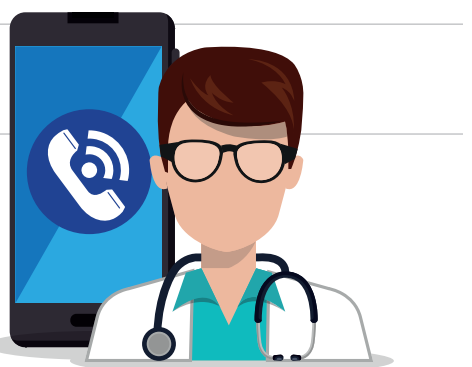

Figura 1. Infografía del nutritionDay Colombia 2021. 


\section{Referencias bibliográficas}

1. Cardenas D, Bermúdez C, Pérez A, Diaz G, Cortés LY, Contreras CP, et al. Are traditional screening tools adequate for monitoring the nutrition risk of in-hospital patients? An analysis of the nutritionDay database. JPEN J Parenter Enteral Nutr. 2021. doi: 10.1002/jpen.2085

2. Cardenas D, Bermúdez C, Pérez A, Diaz G, Cortes LY, Contreras CP, et al. Nutritional risk is associated with an increase of in-hospital mortality and a reduction of being discharged home: Results of the 2009-2015 nutritionDay survey. Clin Nutr ESPEN. 2020;38:138-145. doi: 10.1016/j. clnesp.2020.05.014

3. Cárdenas D, Bermúdez CE, Echeverri S, Pérez A, Puentes M, López L, et al. Declaración de Cartagena. Declaración Internacional sobre el Derecho al Cuidado Nutricional y la Lucha contra la Malnutrición. Nutr Hosp. 2019;36(4):974980. doi: $10.20960 /$ nh.02701 